\title{
A physics-based theory to navigate across risks and opportunities in the performance space: Application to crisis management
}

\author{
Frederick Benaben ${ }^{1 \& 2}$ \\ Matthieu Lauras ${ }^{1 \& 2}$ \\ ${ }^{1}$ Centre Génie Industriel \\ IMT Mines Albi, France \\ (first.last@mines-albi.fr)
}

\author{
Benoit Montreuil ${ }^{2}$ \\ Louis Faugère $^{2}$ \\ ${ }^{2}$ Georgia Institute of \\ Technology, USA \\ (first.last@isye.gatech.edu)
}

\author{
Juanqiong $\mathrm{Gou}^{3}$ \\ Wenxin $\mathrm{Mu}^{3}$ \\ ${ }^{3}$ Beijing Jiaotong University, \\ China \\ (first.last@bjtu.edu.cn)
}

\begin{abstract}
This article presents an original theory for system management, based on physics principles. That theory considers that risks and opportunity can be seen as forces pushing or pulling a system with regards to its objective and its KPIs. Based on that proposal, this article presents the theory, based on (i) identification of susceptibility of systems to internal and external characteristics (danger, favorable conditions), thus creating forces (risks and opportunities), and (ii) evaluation of the sensibility of systems to these forces, thus creating consequences (damages or benefits). This article also presents the practical vision of that theory by detailing the way to observe the force-inducted trajectory of the considered system with regards to its KPIs. An illustrative example and discussions about the perspectives conclude the article.
\end{abstract}

\section{Introduction}

Managing an organization requires to try to reach objectives. These objectives can be of various types but mostly they can be represented by KPIs (this is even the raison d'être of KPIs). Of course, there may be some intangible objectives that couldn't be represented through formal KPIs, but basically, this paper considers that the large majority of objectives can be assessed with formal KPIs. Consequently, the management of an organization is intended to try to bring the organization's KPIs to some predefined targeted values. So, schematically, the management of any organization aims at controlling the trajectory of that organization within the framework of its KPIs.

The way these KPIs evolve is the consequence of changes due to the occurrence of potentialities, which then become actualities. Roughly speaking, if the consequences of an actuality are positive with regards to the targeted KPIs (getting closer), the potentiality is an opportunity. Symmetrically, if the consequences are getting the organization away from the target values for its KPIs, then, the potentiality is a risk.
The position of this article is to claim that the management of an organization requires to identify objectives and to deal with decisions to seize or escape potentialities (opportunities or risks) in order to reach the targeted performance level associated to these objectives. Obviously, there are a lot of principles and definitions hidden behind this vision, but this article is dedicated to try to make them clear by presenting a full theory for the control of an organization's trajectory. The question that this article (and the presented theory) aims at answering is the following: "how could the risks and opportunities related to a considered organization be identified, assessed and analyzed to illuminate the trajectory of the organization?" This article is organized according to the following structure: Section 2 is dedicated to provide an overview and an analysis of existing research works and scientific contributions that could help to answer the previous question. Section 3 describes the physicsbased theory that aims at answering the previously defined question. Section 4 presents a didactical application of that theory to a crisis management usecase. Finally, section 5 presents some conclusions and perspectives.

\section{Related works}

Managing potentialities implies to deal with both opportunities and risks. As presented in [1], opportunities and risks are actually very close and symmetric concepts (pulling or pushing the observed system with regards to its goals). However, the domain of risk management is far more developed and studied than the domain of opportunity management. This is actually not an issue, especially due to their symmetry. Thus this section is mainly focused on contributions and results regarding risk management but mostly, these can be considered as actual regarding opportunity management and more broadly potentialities. 


\subsection{General statements}

There are a few considerations that are nowadays generally admitted in the field of risk management.

First of all, the risk management process is generally described according to three or four phases (sometimes the fourth phase is omitted) as described in [2] and [3]:

- Risk identification

- Risk assessment / evaluation

- Risk mitigation

- Monitoring of the situation

Risk identification is dedicated to study the organization and its environment in order to detect potentialities. Risk assessment (or evaluation) provides some tangible values to the risk. Risk mitigation aims at limiting the bad consequences of the risk (or enhancing the good consequence in the case of an opportunity). The monitoring of the situation aims at supporting the detection feature making this waterfall process a cycle.

Another generally admitted statement concerns the vision of risk. In [4] risk is described as the combination of its probability of occurrence on the one hand and the impact on the organization on the other hand (where the impact can be considered as the consequences on the KPIs of the organization). This vision can of course be extended to the notion of opportunity (with benefit instead of impact) as presented in [5] and recall on Figure 1:

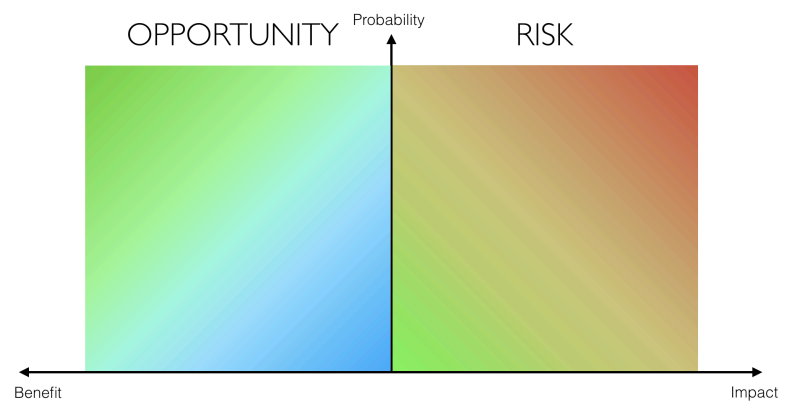

Figure 1. Two dimensions of potentiality.

It is a very classical two dimensions representation of potentiality that is mainly used to visualize and to rank risks in the perspective of mitigation actions.

\subsection{Risk management technics}

There are a lot of well-known and applied risk management technics. From the very complete literature review of [2], we can cite the following ones:

- FMEA: Failure Mode and Effects Analysis presented in [6]. The principle of FMEA is to identify for each component of a system all the possible failure modes and their consequences on the whole system. These consequences are then ranked in a framework similar as the one presented on figure 1.

- FTA (and ETA): Fault Tree Analysis (and Event Tree Analysis) presented in [7]. This method is based on analysts able to model causes (backward with FTA) or effects (forward with ETA) of failures as logic diagrams, to support decision-making.

- HAZOP: Hazard and Operational study, presented in [8]. It is a brainstorming technics based on the use of specific words to introduce limits and detect potential risks. It is mainly used in chemical domain.

- CBA (and RBA): Cost Benefit Analysis (and Risk Benefit Analysis), presented in [9]. This technique is based on compiling a balance sheet of benefits versus costs (or risks).

- SA: Sensitive Analysis, presented in [10]. This is a robustness evaluation approach based on tracing "what if" questions.

- Hertz-type simulation: this simulation approach can be used to generate a probability distribution based on the various combinations as presented in [11].

- Monte Carlo: As presented in [12], in the context of risk management, Monte Carlo can be used to reduce the variance of probability.

- Expert Systems: In the context of risk management, expert systems can be used to infer causal chains as presented in [13].

According to [2], these approaches can be located with regards to the phases of risk management process:

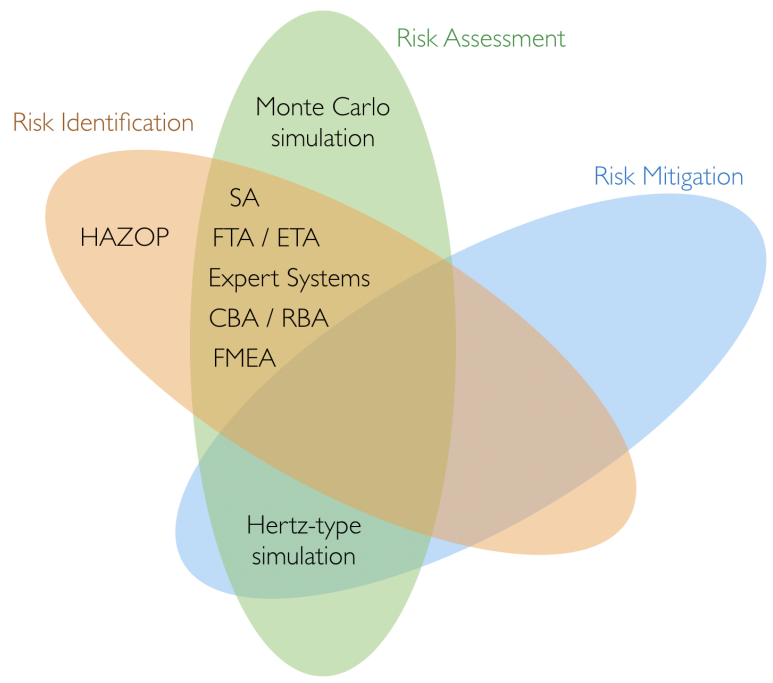

Figure 2. Risk management technics versus phases.

Obviously, and from the previous study, the mitigation phase is not satisfyingly covered. It is quite 
a logical statement due to the fact that identification and assessment are the phases dedicated to prepare the material for decision makers to take their decisions. Mitigation has always been considered more like a human only activity.

\subsection{Contributions from Management Science}

Within the domain of Management Science, and especially in the field of strategic approaches and tools, there are several well-recognized (even if not systematically well-appreciated) tools and approaches. For instance, Porter's five forces analysis, SWOT Analysis, PEST Analysis, Scenario Planning, Value Chain Analysis. In [14] a study of a wide-range of public and private organizations in an economic region of the UK shows that $70 \%$ of these organizations have ever used a SWOT analysis, $28 \%$ for scenario planning, $20 \%$ for value chain analysis, $13 \%$ for Porter's five forces.

The SWOT analysis (originated from Harvard Business School in the 1950s or 1960s) is the essential and indispensable approach to comment. SWOT (or SVOR in project management) stands for Strengths, Weaknesses, Opportunities, and Threats (respectively Strengths, Vulnerabilities, Opportunities, and Risks). It is usually represented as a $2 \times 2$ matrix:

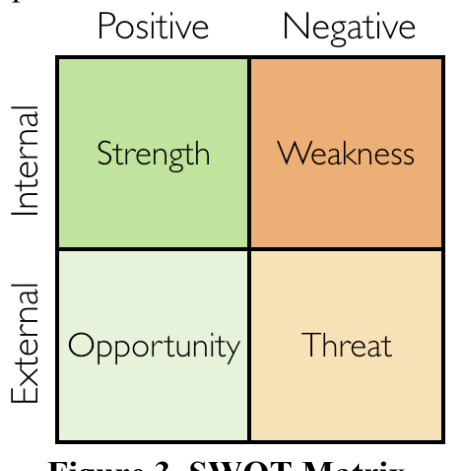

Figure 3. SWOT Matrix.

As presented in [15], the SWOT Analysis is mainly dedicated to the classification of (i) attributes of the considered system (Strengths and Weaknesses) and (ii) external potentialities (Opportunities and Threats).

One interesting aspect of [15] is the comparison of the SWOT analysis with TELESCOPIC (an approach introduced in the same article) which is very close to PEST, except richer. There is a full set of approaches similar to PEST (originated from F. Aguilar in the 1960s at Harvard and successively improved by a series of authors such as A. Brown or T. Davenport) like STEP, PESTE, PESTEL or the already mentioned TELESCOPIC. Basically, all these approaches provide a mnemonic for sectors that should be considered as sources of risks and opportunities. Basically for
PESTEL: P (political), E (Economic), S (social), T (Technological), E (Environmental), L (Legal).

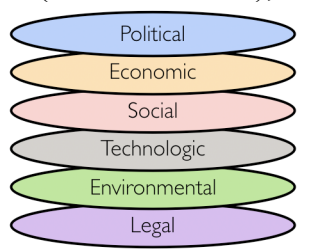

Figure 4. PESTEL sectors.

One interesting connection between SWOT and PESTEL is that the external Opportunities and Threats (basically opportunities and risks) could be identified by investigating the PESTEL sectors.

\subsection{Synthetic vision of potentiality}

From the previous related works, potentiality (risk or opportunity) has to be considered from the dimensions of its probability and of its impact on the considered system (cf. 2.1). Besides, there are manual tools and approaches for potentialities identification (cf. 2.2 and 2.3)

In addition to the previous considerations, [16] describes risk according to three main elements: (i) a danger (or driver) that induces the risk, (ii) an event that embeds the probability of occurrence, and (iii) the consequences representing the actual impact of the occurrence of the risk. A very similar structure has been used in [17] for the description of a causal danger - risk - consequence chain. One very important aspect of that causal chain is that it can be generalized, as presented in [5], to characteristic (danger or favorable condition) - potentiality (risk or opportunity) consequence (damage or benefit). See figure 5:

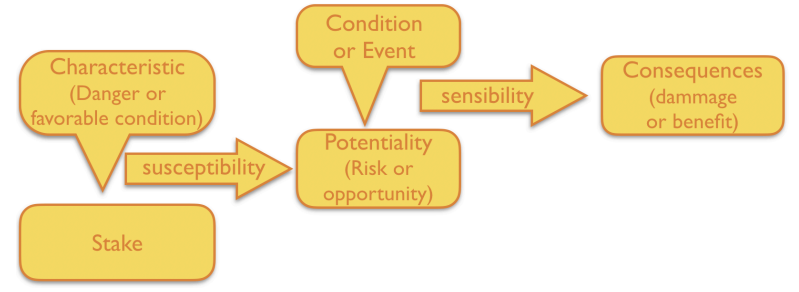

Figure 5. The potentiality causal chain model.

That model is compatible with the classical vision of section 2.1: The probability of a risk is represented through the probability of the condition or event to be true on Figure 5. The impact of a risk is represented by the consequences on figure 5.

Besides, this model also shows that the approaches presented in section 2.2 and 2.3 can be considered as methods to identify characteristics and potentialities of the model of figure 5. Especially, the methods of section 2.2 can be used to identify potentialities that 
can be distributes in the SWOT matrix, while the PESTEL approach can contribute to identify characteristic of the considered system and structure potentialities in the SWOT Matrix. These consistency principles can be described as presented on figure 6 :

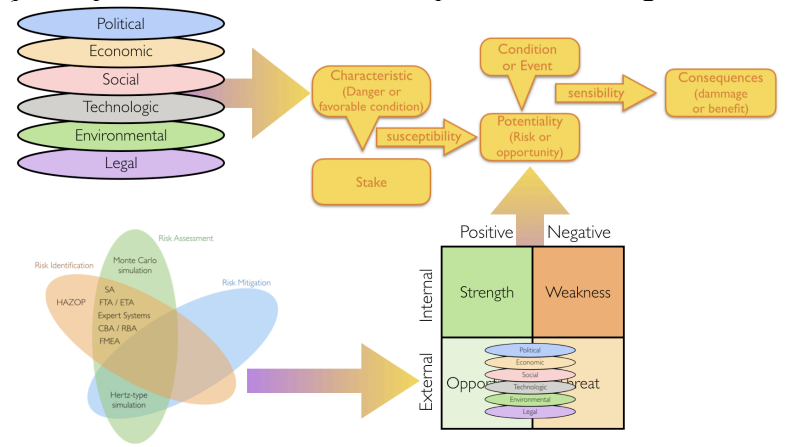

Figure 6. The potentiality model and existing works.

As a conclusion of this section, the existing approaches on risk identification are mainly based on literature review, interviews, and other experiencebased methods ([18] and [19]). As a consequence:

- The classical model for potentiality (impact VS. probability) is difficult to use for cascading effect and anticipation.

- No automated, data-driven tool for potentiality identification has been identified within the previously literature review.

So as an update regarding the initial question presented in the introduction "how could the risks and opportunities related to a considered organization be identified, assessed and analyzed to illuminate the trajectory of the organization?" the next section presents the envisaged approach. This approach focuses on (i) the use of data to continuously model potentialities, and (ii) the use of the potentiality models to support decision-making in the context of risk and opportunity management.

\section{A physics-based theory}

The objective of this section is to describe a potentiality management theory that takes data as input and apply physical and mechanical principles to continuously provide the human users with outputs that support their decision-making processes.

\subsection{Data management framework}

As it has been discussed in [5] and formally described in [20], supporting decision-making can be based on climbing the following abstraction levels: (i) Data, thanks to perception, (ii) Information, thanks to interpretation, and (iii) Decision, thanks to exploitation. Besides, this abstraction rising can be based on the interaction with knowledge such as metamodel or reference base of instance, etc. This principle is illustrated on the next figure 7 :

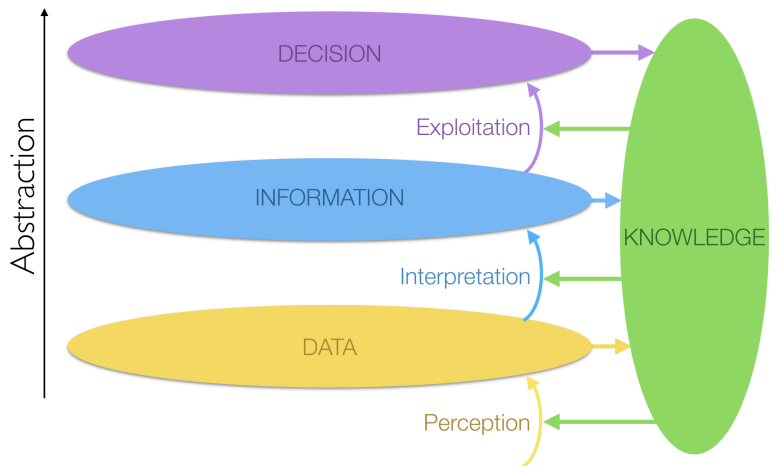

Figure 7. Abstraction rising to reach decision.

In the context of this article, the previous abstraction framework can be instantiated as follow:

1. By collecting data (current and forecast) related to the observed situation, i.e. the considered system and its environment, the approach reaches the first DATA level.

2. By interpreting the collected data with regards to a reference metamodel (basically the one implicitly represented with figure 5, describing the concepts of characteristic, potentialities, consequences, etc. and their relations) in order to build a situation model, the approach reaches the second INFORMATION level.

3. By using the situation model to perform calculation, simulation and other types of model exploitation, especially in order to evaluate the consequences of the identified potentialities (in the situation model), the approach reaches the DECISION level.

In the context of this article, the first step will not be described. The following considers that data (including actual data and forecasts) can be gathered and that this data is significant and trustable. Discovery, trust, cleaning of data is out of the scope of this article. It absolutely does not mean that these steps are obvious, trivial or not relevant (it is quite the opposite), but the focus of this article is based on the hypothesis that clean and usable data can be collected. As a consequence, the following ignores the perception stage and focuses on presenting the interpretation and exploitation steps.

\subsection{Interpretation of Data}

The goal of this step is to use the continuously collected data to build and maintain a situation model. 
This model is based on the following metamodel (formalizing the vision presented in figure 5):

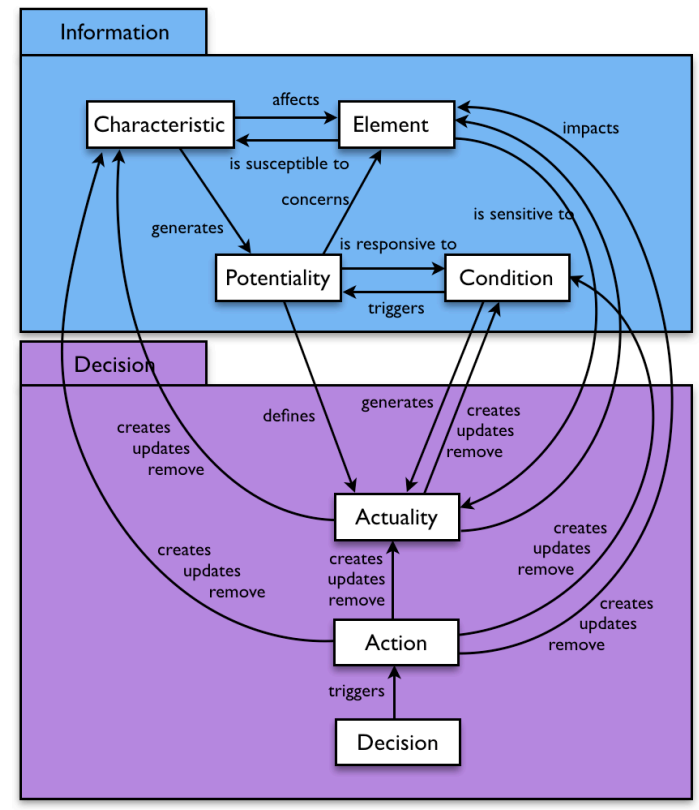

Figure 8. Potentiality management metamodel.

This metamodel presents both the concepts related to the information level and the ones related to the decision level. Basically, the objective of the information level is to interpret data to instantiate concepts of the information package while (one of) the objective(s) of the decision level is to use data and the model of the information level to infer the instance of the decision package. The concepts are:

- Characteristic: danger or favorable condition.

- Element: the considered system (or part of it).

- Potentiality: risk or opportunity for an element.

- Condition: event that triggers the potentiality

- Actuality: consequence of the occurrence of a potentiality.

- Action: Act that can impact actualities, conditions, characteristics and elements.

- Decision: choice that triggers an action.

The question is now: "how do we instantiate the concepts of the information package of the metamodel based on the available data?". Basically, the principle that is used to answer this question is the following:

The gathered data is used to fill in a multidimensional framework. This framework is based on the dimensions that are representative of the considered situation: geographical (latitude, longitude, altitude), type of system/organization (domain, sectors, size), dependency (type of inputs, type of outputs, type of energy), status (legal status, social status), and of course a lot of others dimensions directly depending on the considered domain. The main interest of this modeling space (the susceptibility framework) is that any characteristic of the observed situation can be model as a Boolean value in this susceptibility framework. In addition any element can also be located in this susceptibility framework (depending on its geographical location, its type, its size and more generally its values on all dimensions). It actually means that for each point in space, the considered characteristics affects (1) or not (0) the elements located in that point. Let's take two examples: the seismic characteristic of California (characteristic) would only impact directly elements of any kind with geographical location in California. Similarly, a European project of tax reduction for companies of less than 250 employees based on the number of handicapped employees would only impact directly SMEs located in Europe. Of course, it can be a more sophisticated function than a Boolean (like a distribution between 0 and 1 depending on the strength of the impact, or even between -1 and 1). Figure 9 illustrates that susceptibility framework in a very simple case regarding companies based on their size and location.

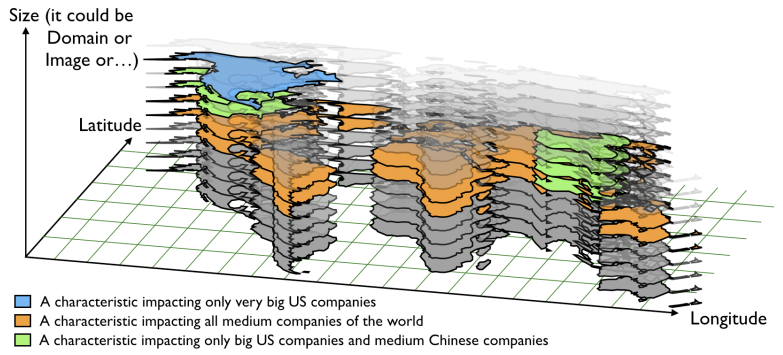

Figure 9. A 3D Susceptibility Framework.

This idea can be represented with the following:

- An element $\mathrm{E}$ can be characterized according to the $\mathrm{N}$ dimensions of the Susceptibility Framework: $\mathrm{E}=\left(\mathrm{e}_{1}, \mathrm{e}_{2}, \ldots \mathrm{e}_{\mathrm{n}}\right)$ such as longitude, latitude, type, nature, size, type of inputs, type of outputs, etc.

- A characteristic of the environment is a Boolean (or a distribution) function of occupation in the Susceptibility Framework: $\mathrm{Ch}\left(\mathrm{x}_{1}, \mathrm{x}_{2}, \ldots \mathrm{x}_{\mathrm{n}}\right)=1$ if the characteristic $\mathrm{Ch}$ is actually present in the location $\left(\mathrm{x}_{1}, \mathrm{x}_{2}, \ldots \mathrm{x}_{\mathrm{n}}\right)$. Else, $\mathrm{Ch}\left(\mathrm{x}_{1}, \mathrm{x}_{2}, \ldots \mathrm{x}_{\mathrm{n}}\right)=0$.

- The location of $\mathrm{E}$ in these $\mathrm{N}$ dimensions determines its susceptibility to characteristics: $\mathrm{E}$ is susceptible to all characteristics that have a non-null value at the coordinates of $E$.

Finally, the principle of the interpretation stage is to use incoming data to locate, on the one hand characteristics, and on the other hand elements in the Susceptibility Framework. Then, based on matching location in that framework, the existence of 
potentialities can be inferred. On a strict point of view, this mechanism does allow to automatically instantiate potentialities but (i) it does not allow to characterize these potentialities (is it a risk or an opportunity? What are its probability and impact?), (ii) it does not allow to identify its triggering condition(s), and (iii) the potentialities only have a possible impact on all the elements with the same location.

So basically, at the end of the Interpretation stage, and depending on the available data flow, the situation model contains the characteristics, elements and potentialities (and their connections) related to the observed situation.

\subsection{Exploitation of Information}

The goal of this step is to use the identified potentialities to create a decision support environment. Basically, this objective can be summarized as: considering the identified potentialities, the goal is to infer the associated latent consequences (actualities).

One of the key idea of this approach is then the following: Considering that opportunities and risks are potentialities that push or pull the considered system with regards to its KPIs (basically a risk would get the system away from the target values of its KPIs while an opportunity would get it closer), let's considered potentialities as forces in the frameworks of the system's KPIs.

Consequently, let us introduce a second framework, this one dedicated to the exploitation transition between information and decision: the Decision Framework. The dimensions of this framework are the significant KPIs of the considered type of systems. If the system is a supply chain, then the dimensions could be cost, quality, and time. If the system is a sport team, then the dimensions could be offensive performance, and defensive performance, etc.

The objective of this framework is to locate the considered system (or elements of the systems) in terms of KPI and to define its net of forces (each force is actually the representation of the probable consequences of each identified potentiality). Then, the aim is to define, by summing and combining these identified forces, the appropriate combination of potentialities to trigger in order to reach the target spot of the Decision Framework. This combination of potentialities corresponds to a set of decisions and actions to take to trigger (or not to trigger) specifically the selected potentialities.

Another benefit is that in addition to seeking for the best combination of potentialities to activate in order to reach the target zone, this framework can also be used to define the target zone itself. By looking at all the identified forces and their intensity, one can study the available combinations (and especially the ones with low cost triggering decisions and actions) to define the best compromise between the improvements of the KPIs on the one hand and the required effort on the other hand. Figure 10 illustrates this principle:

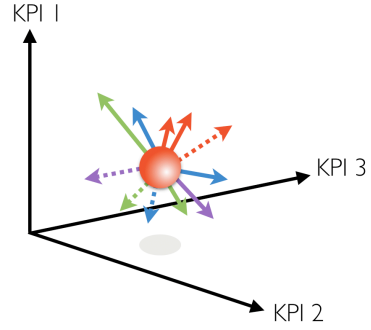

Figure 10. A 3D Decision Framework.

The question now is: "how could the forces be defined, considering that the potentialities have been identified (thanks to the susceptibility detection mechanism) but have not been characterized in terms of potential consequences?". The answer to that question is based on the definition of a protocol of evaluation of the actual impact of a potentiality.

More precisely on this impact, these research works consider that the potentiality might impact the element according to a relative term and an absolute term: the relative term depends on the current location of the element with regards to its KPIs (e.g. $20 \%$ of the profit will be lost, in the case of a KPI on the profit) while the absolute term does not depend on that location (e.g. a net profit of $\$ 10,000$ whatever the current profit).

This idea can be represented with the following:

- If $\mathrm{E}$ is susceptible to a characteristic $\mathrm{Ch}$, then there is a matrix $\mathrm{M}_{\mathrm{Ch}}$ and a vector $\mathrm{V}_{\mathrm{Ch}}$ that represent respectively the relative term and the absolute term of the impact of the characteristic $\mathrm{Ch}$ on $\mathrm{E}$ (i.e. the force of the potentiality generated by $\mathrm{Ch}$ ). Then the force of $\mathrm{Ch}$ on $\mathrm{E}$ is: Force $=\mathrm{M}_{\mathrm{Ch}} \cdot \mathrm{E}+\mathrm{V}_{\mathrm{Ch}}$.

- $\quad \mathrm{M}_{\mathrm{Ch}}$ is a mxm matrix (impact matrix) and $\mathrm{V}_{\mathrm{Ch}}$ is a vector (impact vector) of $m$ terms (where $\mathrm{m}$ is the number of KPIs considered for $\mathrm{E}$ ). If the $\mathrm{m}$ KPIs are independent, then $\mathrm{M}_{\mathrm{Ch}}$ is a diagonal matrix.

Force $=\left[\begin{array}{ccc}m 1,1 & \cdots & m 1, m \\ \vdots & \ddots & \vdots \\ m m, 1 & \cdots & m m, m\end{array}\right] . C+\left[\begin{array}{c}V 1 \\ \vdots \\ V m\end{array}\right]$

- Each term $\mathrm{m}_{\mathrm{i}, \mathrm{j}}$ represents the specific relative impact of $\mathrm{Ch}$ on the $\mathrm{KPI}_{\mathrm{i}}$ due to the value of $\mathrm{KPI}_{\mathrm{j}}$ (if the KPIs are independent, there are only $\mathrm{m}_{\mathrm{i}, \mathrm{i}}$, representing the specific impact of $\mathrm{Ch}$ on the $\mathrm{KPI}_{\mathrm{i}}$ due to its current value). Each term $\mathrm{V}_{\mathrm{i}}$ represents the specific absolute impact of $\mathrm{Ch}$ on the $\mathrm{KPI}_{\mathrm{i}}$. 
- It is interesting to notice that depending on the nature of the considered characteristics, there might be profile of matrix and vectors.

The following question is: "how could the different terms of the Matrix $M_{C h}$ and of the Vector $V_{C h}$ be defined?". There may be several avenues related to data analytics to answer to that question. The current works are based on the notion of micro-consequences ( $\mu$-consequences) as inputs for a simulation:

The principle is to define a simulation model of the observed system dedicated to evaluate the KPIs of that system. The next step is to define (manually or based on historic data) the specific $\mu$-consequences of each potentiality on the overall situation (a $\mu$-consequence could be for instance "between 20\% and 30\% of the delivery trucks will face a 2 to 3 hours delay"). Then, the following step is to pass on these $\mu$-consequences to the input variables of the simulation model and to run the simulation (including the potentially implicit stochasticity). The observed KPI as outputs of the simulation may be different from the initial ones and represent the impact of the considered potentiality (actually, of the $\mu$-consequences of that potentiality) to the considered system. All $\mu$-consequences are used to either assess the actual KPIs value (certain/present) or as inputs of the simulation tool to generate the associated matrix for the expected forces (certain/future) and for the unknown ones (uncertain/ future). Figure 11 represents this principle:

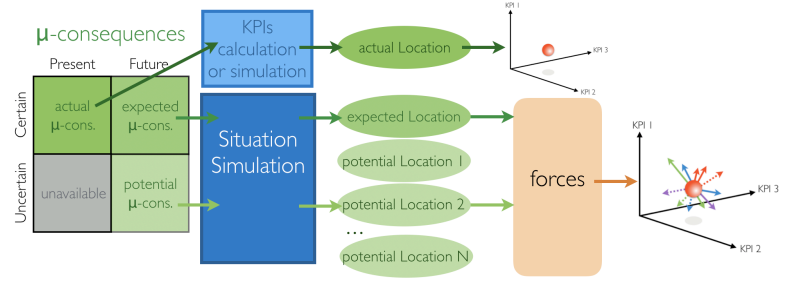

Figure 11. From $\mu$-consequences to forces.

One strong hypothesis in that approach concerns the supposed independence of characteristics and the resulting summability of all the obtained impact Matrix and Vectors (just like the forces are supposed to be summable). It is clear that in some cases this hypothesis is wrong (in such a case, a specific impact should be determined for the combination of dependent potentialities, resulting in a Matrix and a Vector representing the set of $\mu$-consequences associated to the combination of potentialities) but in a lot of cases, the potentialities are impacting very different aspects of the system and can be considered as independent.

\subsection{The obtained forces}

Basically, the obtained forces, in addition to their direction and intensity (within the Decision Framework of the KPIs dimensions) are of different natures and types. These characteristics may be interesting to define patterns of impact matrix and vectors. Figure 12 represents these natures and types of forces:
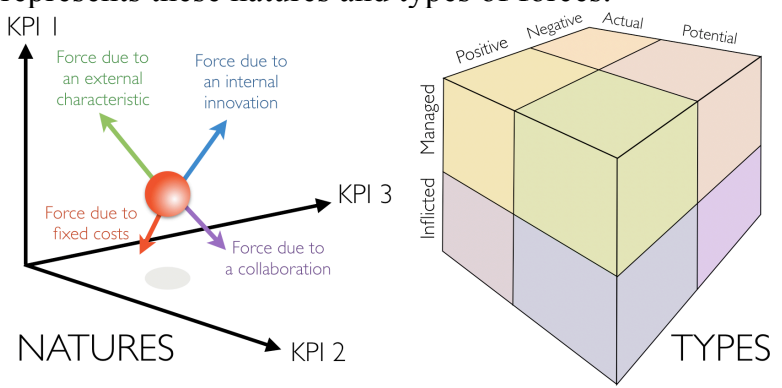

Figure 12. Types and natures of forces.

The four different force natures are the following:

- External field force: the force is due to an external characteristic (weather, new law, seismic area, etc.).

- Collaboration force: the force is due to a collaboration (a supplier or a customer, sharing of a resource, etc.).

- Internal force: the force is due to some internal action (reorganization, buying a resource, etc.).

- Gravity force: the force is due to some unavoidable internal weights (fixed costs, etc.).

The force types are based on the following criteria:

- Is the force actual or potential?

- Is the force positive or negative (is it an opportunity or a risk)?

- Is the force inflicted or managed (is there one of our decisions that can trigger it or not)?

\section{An illustrative example in the domain of crisis management}

Nota Bene: The following example is fictional and even if the data is realistic, the scenario is not. This use-case is dedicated to illustrating the theory.

The Loire river flooding is one of the three most feared disasters in France (with the Seine river flooding and the earthquake in south-east of France). In 1856, such a flood had flooded 110,000 hectares occupied today by more than 300,000 people, 13,600 companies and 72,000 jobs [21]. To enable the simulation of such a scenario, the Loire-Cher-Indre and Maine-Loire Services of Forecast of Floods (In French SPC) did provide a model of propagations: a 100-year flood on the Middle Loire, between the Bec d'Allier and the Bec de Maine. This is the highest known flood event for a given region [22]. This hydrometeorological scenario, reported in [21], realistically models the water inflow of the six tributaries present in 
this part of Loire in the case of a 100-year flood. Thus, three daily flow and water levels forecast were developed for the seven reference hydrometric stations and the 11 expected flood days. Each survey contains forecast water flows and levels over the next 56 hours and three probability levels: high, median and low. In the following, the use-case will focus on the city of Orleans and on the associated hydrometric station.

\subsection{The observed situation}

The considered system is the city of Orleans facing a river flooding. In this example, two characteristics will be considered: (i) the evolution of the behavior of the Loire river, and (ii) the rules and doctrine that concern such a crisis in a French city. Clearly, in that case, the city of Orleans is susceptible to both of them. Noticeably, other characteristics could be relevant (e.g. the local social climate, the period of the year, etc.) but the example will focus on these two.

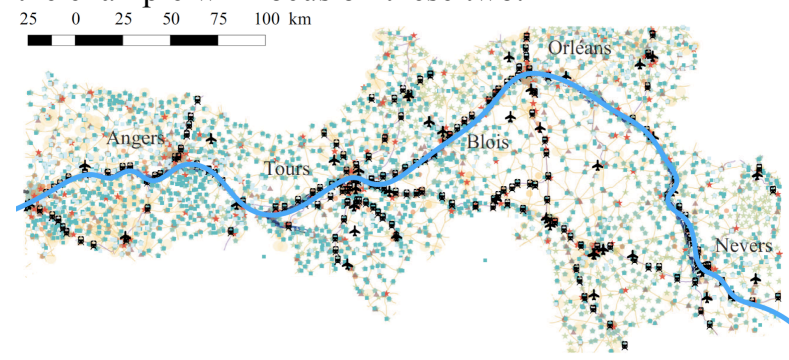

Figure 13. The Loire River and the area of Orleans.

The considered KPI framework is a 3D framework based on the following dimensions: (i) cost, (ii) casualties, and (iii) bad image. Obviously, the objective of the management is to minimalize the KPIs.

Considering that the (two) characteristics have been identified, this example will focus on the exploitation of information (second step). Let's start the scenario at the beginning of the flooding event.

\subsection{Exploitation of Information}

The data that can be considered as source of $\mu$ consequences are: (i) the hydrological forecast related to the behavior of the Loire river, and (ii) the expected consequences of the doctrinal actions (evacuation, opening of the dam, opening of the upstream weir, etc.). Figure 14 presents the official hydrological forecast of water level and water flow in Orleans. The simulation is based on a topographic simulation, estimating, based on the water levels and flows, the submersed surface, the cost of fixing the damages and the average casualties. For the consequences of the doctrinal actions, this article only considers the evacuation. The associated data is the consequences of evacuating Orleans (115,000 people), estimated by the Local Prefecture (Loiret). The consequences on the Bad Image KPI have been manually estimated by the communication cell of the Loire prefecture.

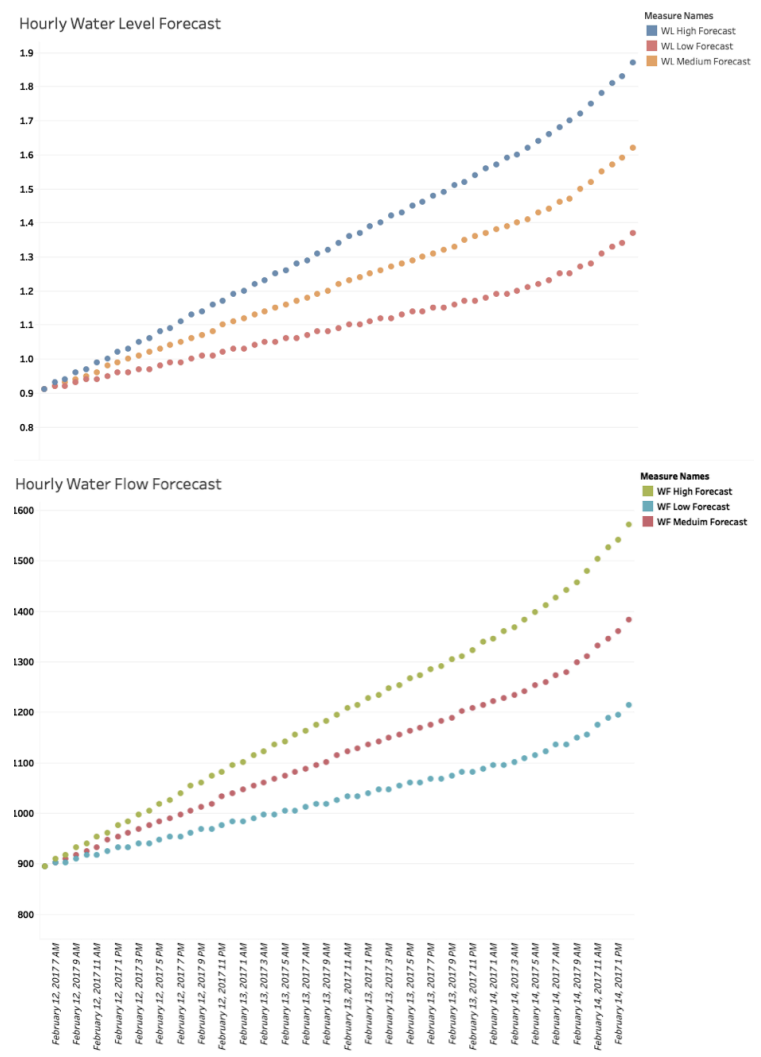

Figure 14. Water Level/Flow (high, medium, low).

At the very first stage of the crisis, there are factual values for the Loire River flow and level, and the only potentiality is about the evacuation: the initial obtained matrix and vectors are the following:

- Evacuation:

Fevac $=\left[\begin{array}{ccc}1 & 0 & 0 \\ 0 & 1.5 & 0 \\ 0 & 0 & 2\end{array}\right] . E+\left[\begin{array}{l}3 \\ 0 \\ 3\end{array}\right]$ with $E=\left[\begin{array}{c}E \$ \\ \text { Ecas. } \\ \text { Eima. }\end{array}\right]$

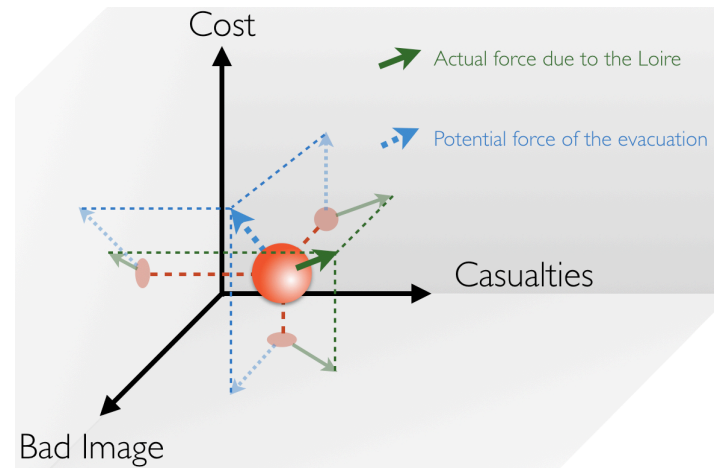

Figure 15. Initial position and forces. 
For clarity reason, the scales have been normalized. As shown on figure 15, the evacuation force and the Loire force are not pushing Orleans in the same way.

The evacuation force is a potential, managed force (cf. figure 12). If the decision of evacuation is not taken, Orleans may move in the framework (because of the Loire force) and there will be new forces associated to that new position (see figure 16). The matrix and vectors of that new position are the following:

- Behavior of the Loire River HIGH:

FLoire $+=\left[\begin{array}{lll}1 & 0 & 0 \\ 0 & 3 & 0 \\ 0 & 0 & 2\end{array}\right] . E+\left[\begin{array}{l}4 \\ 4 \\ 2\end{array}\right]$

- Behavior of the Loire River MEDIUM:

FLoire $=\left[\begin{array}{ccc}1 & 0 & 0 \\ 0 & 2 & 0 \\ 0 & 0 & 1.5\end{array}\right] . E+\left[\begin{array}{c}2 \\ 2 \\ 1.5\end{array}\right]$

- Behavior of the Loire River LOW:

FLoire $-=\left[\begin{array}{ccc}1 & 0 & 0 \\ 0 & 1.5 & 0 \\ 0 & 0 & 1.2\end{array}\right] . E+\left[\begin{array}{l}1 \\ 1 \\ 1\end{array}\right]$

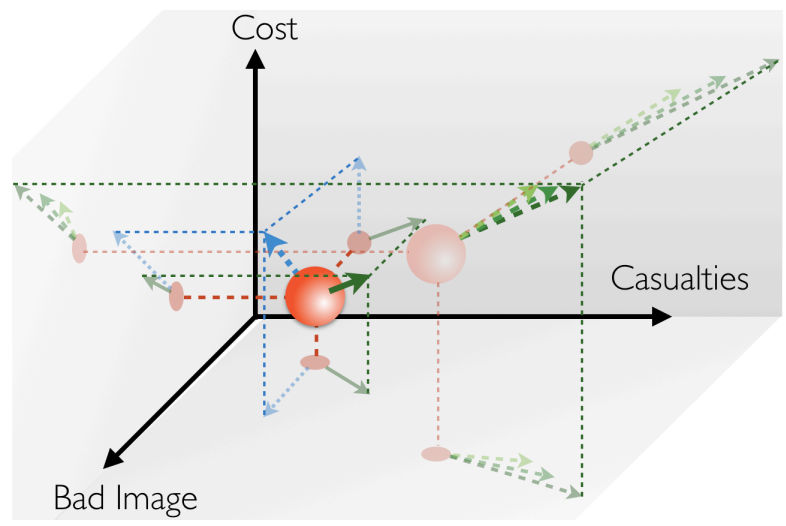

Figure 16. Possible position and forces of Orleans if no evacuation decision has been taken.

On figure 16, the light red sphere represents the potential position of Orleans, pushed by the Loire force only (after one time unit). As a consequence of that new position, three potential forces due to the Loire River have been calculated (green vectors) that represent a strong stream for Orleans in its KPIs framework (note that the new vector for the evacuation has not been calculated).

On the contrary if the evacuation decision is taken, Orleans may move in the framework (because of the evacuation force) and there will be new forces associated to that new position (see figure 17). See below the associated matrix and vectors:
- Behavior of the Loire River HIGH:

FLoire $+=\left[\begin{array}{ccc}1 & 0 & 0 \\ 0 & 1 & 0 \\ 0 & 0 & 0.6\end{array}\right] . E+\left[\begin{array}{l}2 \\ 0 \\ 0\end{array}\right]$

- Behavior of the Loire River MEDIUM:

FLoire $\sim=\left[\begin{array}{ccc}1 & 0 & 0 \\ 0 & 1 & 0 \\ 0 & 0 & 0.7\end{array}\right] . E+\left[\begin{array}{c}1.5 \\ 0 \\ 0\end{array}\right]$

- Behavior of the Loire River LOW:

FLoire $-=\left[\begin{array}{ccc}1 & 0 & 0 \\ 0 & 1 & 0 \\ 0 & 0 & 0.8\end{array}\right] . E+\left[\begin{array}{l}1 \\ 0 \\ 0\end{array}\right]$

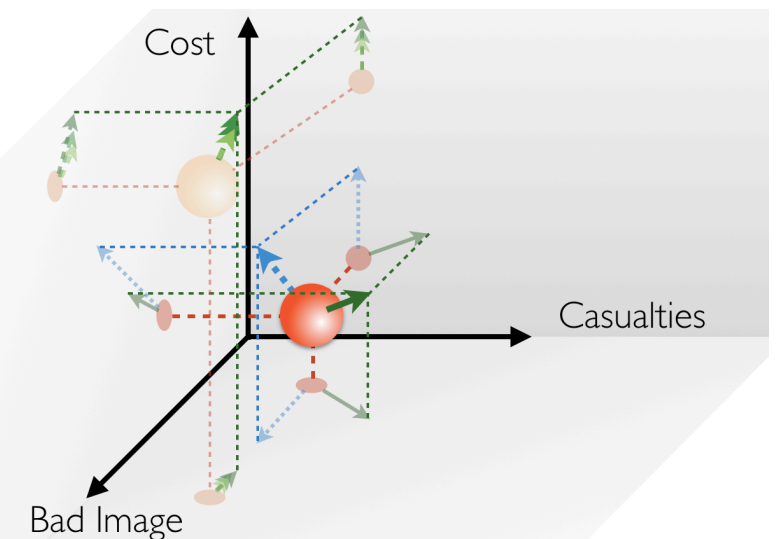

Figure 17. Possible position and forces of Orleans if the evacuation decision has been taken.

On figure 17, the light red sphere represents the potential position of Orleans, pushed by the evacuation force and the initial Loire force (after one time unit). As a consequence of that new position, three potential forces due to the Loire River have been calculated (green vectors) that represent a far more manageable stream for Orleans in its KPIs framework.

\section{Conclusion and perspectives}

As stated by [23] and [24], instability is the norm. There is no rational expectation to maintain any organization in a stable state. However, dealing with instability is the new perspective of management. The theory presented and illustrated in this paper aims at offering an innovative vision for such a purpose. By describing a path between data and decision, based on a physics-based theory, this article claims that it opens the door to a brand new vision of management.

Through that open door, perspectives like "energy reasoning" or "solid mechanics" can be seen. The first one might be a way to deal (i) with impulsion in complement to forces, and (ii) with the valuation of options (in terms of costs and energy). The second may 
be an avenue to deal with the chosen or inflicted deformations of an organization (enterprise, supplychain, or others) to enhance or adapt the faced forces.

Of course, there are still a lot to do to turn that theory into an actionable and workable practice: the trustable and efficient connection with heterogeneous data (from all types of sources), the continuous and (close to) real-time calculation of trajectories, and the visualization. This last aspect of visualization is of the greatest importance considering that the diagrams of the simple use-case of section 4 are already hard to manage (and it is only 3D...). Some research works on the usage of Virtual Reality as a support for visualization of abstract notions have been initiated.

\section{References}

[1] R. Olsson, "In Search of Opportunity Management: Is the Risk Management Process Enough?", International Journal of Project Management, 25(8), pp. 745-752, 2007.

[2] D. White "Application of systems thinking to risk management: a review of the literature", Management Decision, 1995, 33(10), pp.35-45,

[3] W. Ho, T. Zheng, H. Yildiz, and S. Talluri, "Supply chain risk management: a literature review", International Journal of Production Research, Taylor\&Francis, 2015, 53(16), pp. 5031-5069.

[4] P. J. Edwards, P. A. and Bowen, Risk management in project organisations, Elsevier, Oxford, UK, 2005.

[5] F. Benaben, B. Montreuil, J. Gou, J. Li, M. Lauras, I. Koura, W. Mu, "A tentative framework for risk and opportunity detection in a collaborative environment based on data interpretation" Proceedings of 52nd HICSS'19, 2019, Hawaii, USA.

[6] J.J Clifton, "Hazard prediction", in Keller, A.Z. and Wilson, H.C. (Eds), Disaster Prevention, Planning and Limitation, Disaster Prevention and Limitation Unit, University of Bradford and The British Library, 1990.

[7] T. Bell, "Managing risk in large complex systems special report", Spectrum, June 1989.

[8] E.C. Hambly, and E.A. Hambly, "Risk evaluation and realism, proceedings of the Institution of Civil Engineers", Civil Engineering, 102(2), pp. 64-71, 1994.

[9] L.B. Lave, "Approaches to risk management: a critique", Risk Evaluation and Management, Plenum Press, New York, NY, 1986.

[10] V.T. Covello, "Decision analysis and risk management decision making: issues and methods", Risk Analysis, 7(2), pp. 131-139, 1987.
[11] S.S.M. Ho, and R.H. Pike, "The use of risk analysis techniques in capital investment appraisal", in Ansell, J. and Wharton, F. (Eds), Risk: Analysis, Assessment and Management, John Wiley \& Sons, Chichester, 1992.

[12] V.T. Covello, and M.W. Merkhofer, "Risk Assessment Methods - Approaches for Assessing Health and Environmental Risks", Plenum Press, New York, NY,

[13] T.A. Kletz, "Critical Aspects of Safety and Loss Prevention”, Butterworths, London, 1990.

[14] R. Gunn, and W. Williams, W. "Strategic tools: an empirical investigation into strategy in practice in the UK". Strategic Change, 16(5), pp. 201-216, 2007.

[15] G. Panagiotou, "Bringing SWOT into focus" Business strategy review, 14(2), pp. 8-10, 2003.

[16] B. Zeng, P. C. Yen, "Rethinking the role of partnerships in global supply chains: a risk-based perspective", International Journal of Production Economics, 185, pp. 52$62,2017$.

[17] F. Benaben, A.-M. Barthe-Delanoë, M. Lauras, S. Truptil, "Collaborative Systems in Crisis Management: A Proposal for a Conceptual Framework", Proceedings of ProVE'14, Springer, IFIP, Amsterdam, Netherlands, 434, pp. 396-405, 2014.

[18] C. Fang, F. Marle, E. Zio, J. C. Bocquet, "Network theory-based analysis of risk interactions in large engineering projects", Reliability Engineering \& System Safety, 106(2), pp. 1-10, 2012.

[19] X. Zhang, Y. Yang, J. Su, "Risk identification and evaluation of customer collaboration in product development", Journal of Industrial Engineering and Management, 8(3), pp. 928-942, 2015.

[20] F. Benaben, M. Lauras, A. Fertier, N. Salatge, "Integrating model-driven engineering as the next challenge for artificial intelligence - Application to risk and crisis management", Proceedings of the 2019 Winter Simulation Conference, Washington, 2019.

[21] H. Dolidon, "Description of the Loire River Flooding" Deliverable of the French funded project SANCTUM, 2019.

[22] French Ministry of Ecology and Sustainable Development. "Les inondations". Technical report, 2004.

[23] N. Taleb, The Black Swan - The Impact of the Highly Improbable. Random House, New York, 2007.

[24] J. P. Ribeiro, A. Barbosa-Povoa, "Supply Chain Resilience: Definitions and quantitative modelling approaches-A literature review", Computers \& Indus. Engineer., vol. 115, pp. 109-122, 2018. 